\title{
An elementary description of the Mathieu dual hyperoval and its splitness
}

\author{
Satoshi Yoshiara
}

\begin{abstract}
An elementary new construction of a 3-dimensional dual hyperoval $\mathcal{M}$ over $\mathbb{F}_{4}$ is given, as well as an explicit analysis of the structure of its automorphism group. This provides a self-contained introduction to the Mathieu simple group $M_{22}$. The basic properties of $\mathcal{M}$ as a dimensional dual hyperoval, e.g. splitness, complements, linear systems, quotients and coverings, are derived from this construction.
\end{abstract}

Keywords: dimensional dual hyperoval (DHO), the Mathieu DHO, Mathieu group $M_{22}$, automorphism group, complement, linear system.

MSC 2010: 20B25, 20D08, 05B25, 05E18, 51A45, 51E20

\section{Introduction}

The purpose of this paper is to derive basic properties such as the automorphism group, complements and related DHO-sets, substructures, coverings and quotients, of a geometric structure $\mathcal{M}$, called the Mathieu dual hyperoval [9], based on its new simple construction. Specifically, this contains an elementary and self-contained introduction to the Mathieu simple group $M_{22}$ of degree 22, because $M_{22}$ appears as a quotient group of the linear part of the automorphism group of $\mathcal{M}$.

As far as the author knows, the existence of $\mathcal{M}$ was first observed by Jónsson and McKay in terms of the Leech lattice [4, Claim (2.13)], which was explicitly given in a table form using the MOG arrangement [1]. Nakagawa gave an account how to obtain this table, assuming that there exists a certain unitary dual hyperoval [6]. Several characterizations of this structure as dual hyperovals are available (e.g. $[2,5,6])$. 



\section{A simple model of the Mathieu DHO}

\subsection{Notation and review about the exterior product}

In this section, we shall use the letters $i, j$ and $k$ to denote indices in $\{0,1,2\}$ unless otherwise stated. Given an index $i$ in $\{0,1,2\}$, we adopt the convention that

the letters $j$ and $k$ denote the remaining indices, so that $\{i, j, k\}=\{0,1,2\}$.

For example, $\sum_{i=0}^{2} x_{j} x_{k} e_{i}$ denotes $x_{1} x_{2} e_{0}+x_{2} x_{0} e_{1}+x_{0} x_{1} e_{2}$, and so on.

Let $V$ be a vector space of dimension 3 over $\mathbb{F}_{4}$ with basis $e_{0}, e_{1}, e_{2}$. For a vector $x \in V$ and an index $i$ in $\{0,1,2\}$, the letter $x_{i}$ denotes the coefficient of $e_{i}$ in the expression $x=x_{0} e_{0}+x_{1} e_{1}+x_{2} e_{2}\left(x_{0}, x_{1}, x_{2} \in \mathbb{F}_{4}\right)$. We define a nondegenerate symmetric bilinear form on $V$, the dot product, by $x \cdot y:=$ $\sum_{i=0}^{2} x_{i} y_{i}$. For $\alpha \in \mathbb{F}_{4}$, we set $\bar{\alpha}:=\alpha^{2}$. Throughout the paper, $\omega$ denotes a primitive element of $\mathbb{F}_{4}$.

We denote by $S^{2}(V)$ the square symmetric tensor product of $V$, i.e., the factor space $(V \otimes V) / W$ of $V \otimes V$ by the subspace $W$ spanned by $x \otimes y+y \otimes x$ for all $x, y \in V$. The element $(x \otimes y)+W$ in $S^{2}(V)$ with $x, y \in V$ is denoted by $x \otimes y$ for short. (This notation should not cause any confusion, because we only work with $S^{2}(V)$ later.) For $x \in V$, we use the symbol $\Delta(x)$ to denote a vector $x \otimes x$ in $S^{2}(V)$. Notice that $\Delta(x+y)=\Delta(x)+\Delta(y)$ and $\Delta(\alpha x)=\bar{\alpha} \Delta(x)$ for $x, y \in V, \alpha \in \mathbb{F}_{4}$. We adopt the following vectors as a basis for $S^{2}(V)$ :

$$
\Delta_{i}:=\Delta\left(e_{i}\right) \text { and } \nabla_{i}:=e_{j} \otimes e_{k} \text { for } i \in\{0,1,2\},
$$

where we follow the convention above. Explicitly, $\nabla_{0}:=e_{1} \otimes e_{2}, \nabla_{1}:=e_{2} \otimes e_{0}$ and $\nabla_{2}:=e_{0} \otimes e_{1}$. For a vector $v=\sum_{i=0}^{2} v_{i} \Delta_{i}+\sum_{i=0}^{2} v_{i}^{\prime} \nabla_{i}$ in $S^{2}(V)$, the terms $v_{i} \Delta_{i}$ and $v_{i}^{\prime} \nabla_{i}(i=0,1,2)$ are respectively called diagonal and off-diagonal components of $v$.

We define a non-degenerate unitary form $(,$,$) on S^{2}(V)$ as follows: define on the basis $\left(\Delta_{i}, \Delta_{j}\right)=\left(\nabla_{i}, \nabla_{j}\right)=0$ for all $i, j \in\{0,1,2\}$, and $\left(\Delta_{i}, \nabla_{j}\right)=1$ or 0 according as $i=j$ or not, and extend it semi-linearly on $S^{2}(V)$.

Now we introduce a map $\iota$ on $V$ which is so-called quadratic, in the sense that the associated map sending $(x, y) \in V \times V$ to $\iota(x+y)+\iota(x)+\iota(y)(\in V)$ is an (alternating) bi-additive map (in fact, bilinear over $\mathbb{F}_{4}$ ). For $x=\sum_{i=0}^{2} x_{i} e_{i} \in V$, we define

$$
\iota(x):=\sum_{i=0}^{2} x_{j} x_{k} e_{i}=x_{1} x_{2} e_{0}+x_{2} x_{0} e_{1}+x_{0} x_{1} e_{2},
$$








\subsection{Automorphisms}

The construction in the previous section 2.2 makes easy to find explicit shapes of automorphisms, especially those stabilizing the member $A$. In this section, we shall determine the linear part of the automorphism group of $\mathcal{M}$. Recall that an automorphism (resp. linear automorphism) of $\mathcal{M}$ is a semi-linear (resp. linear) bijection on $S^{2}(V)$ which permutes the members of $\mathcal{M}$. We denote by $\operatorname{Aut}(\mathcal{M})$ the group of all automorphisms of $\mathcal{M}$, in which $L(\mathcal{M})$ denotes the subgroup of all linear automorphisms of $\mathcal{M}$. We shall denote by $x \lambda$ the image of a vector $x$ in a vector space $W\left(V\right.$ or $\left.S^{2}(V)\right)$ by a linear map $L$ on $W$, instead of the usual notation $x^{L}$ or $L(x)$. This convention will be adopted throughout the remaining part of this paper.

The following result was obtained in [6] as well, but a proof based on the presentation of $\mathcal{M}$ in Section 2.2 is given here:

Lemma 2.3. Every linear automorphism $\lambda$ of $\mathcal{M}$ preserves the unitary form (, ) on $S^{2}(V):(u \lambda, v \lambda)=(u, v)$ for all $u, v \in S^{2}(V)$.

Proof. A member $A \lambda$ of $\mathcal{M}$ is totally isotropic by Proposition 2.2(iv). In particular, $\left(\Delta_{i} \lambda, \Delta_{j} \lambda\right)=0$ for all $i, j \in\{0,1,2\}$. As $\Delta_{i}=m\left(e_{i}, e_{i}\right), \nabla_{j}=e_{i} \otimes$ $e_{k}=m\left(e_{i}, e_{k}\right)$ and $\nabla_{k}=m\left(e_{i}, e_{j}\right)$ are contained in a member $A\left[e_{i}\right]$ for $i \in$ $\{0,1,2\}=\{i, j, k\}$, we have $\left(\Delta_{i} \lambda, \nabla_{j} \lambda\right)=0$ and $\left(\nabla_{j} \lambda, \nabla_{k} \lambda\right)=0$ inside a totally isotropic space $A\left[e_{i}\right] \lambda$ for any pairwise distinct indices $i, j, k$ in $\{0,1,2\}$. Thus $\left(\Delta_{i} \lambda, \nabla_{i} \lambda\right)=: \gamma_{i}\left(\in \mathbb{F}_{4}\right)$ is not zero by the non-degeneracy of $($,$) .$

Take any $i, j, k$ with $\{i, j, k\}=\{0,1,2\}$. As $\iota\left(e_{i} \times\left(e_{j}+e_{k}\right)\right)=\iota\left(e_{k}+e_{j}\right)=e_{i}$ and $\iota\left(e_{k} \times\left(e_{j}+e_{k}\right)\right)=\iota\left(e_{i}\right)=0$, we have $m\left(e_{i}, e_{j}+e_{k}\right)=e_{i} \otimes\left(e_{j}+e_{k}\right)+\Delta\left(e_{i}\right)=$ $\Delta_{i}+\nabla_{j}+\nabla_{k}$ and $m\left(e_{k}, e_{j}+e_{k}\right)=e_{k} \otimes\left(e_{j}+e_{k}\right)+\Delta(0)=\nabla_{i}+\Delta_{k}$. Inside a totally isotropic subspace $A\left[e_{j}+e_{k}\right] \lambda$, we have $0=\left(m\left(e_{i}, e_{j}+e_{k}\right) \lambda, m\left(e_{i}, e_{j}+e_{k}\right) \lambda\right)$, which is also calculated as $\left(\Delta_{i} \lambda+\nabla_{j} \lambda+\nabla_{k} \lambda, \nabla_{i} \lambda+\Delta_{k} \lambda\right)=\gamma_{i}+\overline{\gamma_{k}}$. As this holds for all $i, j, k$ with $\{i, j, k\}=\{0,1,2\}$, we have $\gamma_{0}=\gamma_{1}=\gamma_{2} \in \mathbb{F}_{2}$, and therefore $\gamma_{0}=\gamma_{1}=\gamma_{2}=1$. Hence $\lambda$ preserves the unitary form $($,$) .$

Before determining the stabilizer of $A$ in $L(\mathcal{M})$, we shall show a result which can be obtained by iterating use of equation (3).

Lemma 2.4. Let $u_{i}(i \in\{0,1,2\})$ be a basis for $V$. For vectors $x=\sum_{i=0}^{2} x_{i} u_{i}$ and $y=\sum_{i=0}^{2} y_{i} u_{i}$ in $V$ with $x_{i}, y_{i} \in \mathbb{F}_{4}(i \in\{0,1,2\})$, we have the following formula, where $\delta$ denotes the determinant of the matrix $r\left(u_{0}, u_{1}, u_{2}\right)$ (see equation (4)) and the indices $i, j, k$ follow the convention $\{0,1,2\}=\{i, j, k\}$ :

$$
m(x, y)=\sum_{i=0}^{2}(x \times y)_{i} m\left(u_{j}, u_{k}\right)+\sum_{i=0}^{2}\left\{x_{i} y_{i}+\bar{\delta} \overline{(x \times y)_{j}(x \times y)_{k}}\right\} \Delta\left(u_{i}\right) .
$$


Proof. As $x \times y=\sum_{i=0}^{2}\left(x_{j} y_{k}+x_{k} y_{j}\right)\left(u_{j} \times u_{k}\right)=\sum_{i=0}^{2}(x \times y)_{i}\left(u_{j} \times u_{k}\right)$, it follows from repeatedly using equation (3) that $\iota(x \times y)$ is the sum of the terms $\sum_{i=0}^{2} \iota\left((x \times y)_{i}\left(u_{j} \times u_{k}\right)\right)$ and $\sum_{i=0}^{2}\left\{(x \times y)_{j}\left(u_{i} \times u_{k}\right) \times(x \times y)_{k}\left(u_{i} \times u_{j}\right)\right\}$. Notice that $\iota(\alpha x)=\bar{\alpha} \iota(x)\left(\alpha \in \mathbb{F}_{4}, x \in V\right)$ and

$$
\left(u_{i} \times u_{k}\right) \times\left(u_{i} \times u_{j}\right)=\left\{\left(u_{i} \times u_{k}\right) \cdot u_{j}\right\} u_{i}+\left\{\left(u_{i} \times u_{k}\right) \cdot u_{i}\right\} u_{j}=\delta u_{i}
$$

by equations (4) and (5). Rewriting the above terms with these remarks, we have $\iota(x \times y)=\sum_{i=0}^{2} \overline{(x \times y)_{i}} \iota\left(u_{j} \times u_{k}\right)+\delta\left\{\sum_{i=0}^{2}(x \times y)_{j}(x \times y)_{k} u_{i}\right\}$. Then $m(x, y)=x \otimes y+\Delta(\iota(x \times y))$ is equal to the sum of $\sum_{i=0}^{2}(x \times y)_{i}\left(u_{j} \otimes u_{k}\right)+$ $\sum_{i=0}^{2} x_{i} y_{i} \Delta\left(u_{i}\right)$ and $\sum_{i=0}^{2}(x \times y)_{i} \Delta\left(\iota\left(u_{j} \times u_{k}\right)\right)+\bar{\delta} \sum_{i=0}^{2} \frac{i=0}{(x \times y)_{j}(x \times y)_{k}} \Delta\left(u_{i}\right)$, which coincides with

$$
\sum_{i=0}^{2}(x \times y)_{i} m\left(u_{j}, u_{k}\right)+\sum_{i=0}^{2}\left\{x_{i} y_{i}+\bar{\delta} \overline{(x \times y)_{j}(x \times y)_{k}}\right\} \Delta\left(u_{i}\right) .
$$

Proposition 2.5. The stabilizer of the member $A$ in the linear automorphism group $L(\mathcal{M})$ coincides with the group $\{\tilde{g} \mid g \in \mathrm{SL}(V)\}$ isomorphic to $\mathrm{SL}(V)$, where $\tilde{g}$ is a bijective linear transformation on $S^{2}(V)$ defined as follows for each $g \in \mathrm{SL}(V)$ with $g_{a}:=e_{a} g=\sum_{i=0}^{2} g_{a i} e_{i}\left(g_{a i} \in \mathbb{F}_{4}\right)$ for $a \in\{0,1,2\}$. (We also follow the convention on indices $\{i, j, k\}=\{a, b, c\}=\{0,1,2\})$ :

$$
\begin{aligned}
\Delta_{a} \tilde{g} & =\sum_{i=0}^{2} \overline{g_{a i}} \Delta_{i} \quad(a \in\{0,1,2\}), \\
\nabla_{c} \tilde{g} & =\sum_{i=0}^{2}\left\{g_{a i} g_{b i}+\overline{\left(g_{a} \times g_{b}\right)_{j}\left(g_{a} \times g_{b}\right)_{k}}\right\} \Delta_{i}+\sum_{i=0}^{2}\left(g_{a} \times g_{b}\right)_{i} \nabla_{i} \\
& =g_{a} \otimes g_{b}+\Delta\left(\iota\left(g_{a} \times g_{b}\right)\right) .
\end{aligned}
$$

Furthermore, $m(x, y) \tilde{g}=m(x g, y g)$ and thus $A[x] \tilde{g}=A[x g]$ for any $x, y \in V$.

Proof. Take any linear automorphism $\lambda$ of $\mathcal{M}$ stabilizing $A$. Then there is a bijection $g$ on $V$ such that $\Delta(x) \lambda=\Delta(x g)$ for every $x \in V$. As $\lambda$ is linear on $S^{2}(V)$, we have $\Delta\left(\left(x+x^{\prime}\right) g\right)=\Delta\left(x+x^{\prime}\right) \lambda=\Delta(x) \lambda+\Delta\left(x^{\prime}\right) \lambda=\Delta\left(x g+x^{\prime} g\right)$ and $\Delta((\alpha x) g)=\Delta(\alpha x) \lambda=(\bar{\alpha} \Delta(x)) \lambda=\bar{\alpha}(\Delta(x) \lambda)=\bar{\alpha} \Delta(x g)=\Delta(\alpha(x g))$ for all $x, x^{\prime} \in V$ and $\alpha \in \mathbb{F}_{4}$, whence $g$ is linear on $V$.

The subsequent arguments are based on the following idea. Observe that for each nonzero vector $x$ in $V, \lambda$ sends the unique member $A[x]$ in $\mathcal{M} \backslash\{A\}$ containing $\Delta(x)$ to the unique member $A[x g]$ in $\mathcal{M} \backslash\{A\}$ containing $\Delta(x) \lambda=$ $\Delta(x g)$. Then $(A[x] \cap A[y]) \lambda=A[x] \lambda \cap A[y] \lambda=A[x g] \cap A[y g]$ for distinct projective points $[x]$ and $[y]$ of $\mathbf{P G}(V)$, and therefore a vector $m(x, y)$ spanning $A[x] \cap A[y]$ is sent by $\lambda$ to a scalar multiple of $m(x g, y g)$ spanning $A[x g] \cap A[y g]$. 

by the action of $\lambda$ on the basis $\Delta_{i}, \nabla_{i}(i \in\{0,1,2\})$. On the other hand, as $g_{i}$ $(i \in\{0,1,2\}$ ) form a basis for $V$, Lemma 2.4 implies that $m(x g, y g)$ equals

$$
\begin{aligned}
& m\left(\sum_{i=0}^{2} x_{i} g_{i}, \sum_{i=0}^{2} y_{i} g_{i}\right) \\
& \quad=\sum_{i=0}^{2}(x \times y)_{i} m\left(g_{j}, g_{k}\right)+\sum_{i=0}^{2}\left\{x_{i} y_{i}+\bar{\delta} \overline{(x \times y)_{j}(x \times y)_{k}}\right\} \Delta\left(g_{i}\right)
\end{aligned}
$$

with $\delta=\operatorname{det}\left(r\left(g_{0}, g_{1}, g_{2}\right)\right)=\operatorname{det}(g)$. Now recall that $m(x, y) \lambda=\gamma_{x, y} m(x g, y g)$ for some nonzero element $\gamma_{x, y}$ in $\mathbb{F}_{4}$, because $(A[x] \cap A[y]) \lambda=A[x g] \cap A[y g]$ and $m(x, y)$ and $m(x g, y g)$ respectively span $A[x] \cap A[y]$ and $A[x g] \cap A[y g]$.

As $\Delta\left(g_{i}\right)$ and $m\left(g_{j}, g_{k}\right)(i \in\{0,1,2\}=\{i, j, k\})$ form a basis for $S^{2}(V)$, this equation with equation (17) and equation (18) implies that

$$
\begin{aligned}
\overline{\operatorname{det}(g)}(x \times y)_{i} & =\gamma_{x y}(x \times y)_{i}, \quad \text { and } \\
x_{i} y_{i}+\overline{(x \times y)_{j}(x \times y)_{k}} & =\gamma_{x y}\left\{x_{i} y_{i}+\overline{\operatorname{det}(g)(x \times y)_{j}(x \times y)_{k}}\right\}
\end{aligned}
$$

for all $i \in\{0,1,2\}$. As $[x] \neq[y]$, there exists $i \in\{0,1,2\}$ with $(x \times y)_{i} \neq 0$. Thus we have $\gamma_{x y}=\overline{\operatorname{det}(g)}$ from the first equation above. Then the second equation above reads that for all $i \in\{0,1,2\}$ we have

$$
\left(x_{i} y_{i}\right)(1+\overline{\operatorname{det}(g)})=(1+\operatorname{det}(g)) \overline{(x \times y)_{j}(x \times y)_{k}} .
$$

Recall that this conclusion holds for every distinct projective points $[x]$ and $[y]$ of $\mathbf{P G}(V)$. Take $x=e_{0}+e_{1}+e_{2}$ and $y=e_{0}+\omega e_{1}+\bar{\omega} e_{2}$. Then we can verify that $x \times y=y$, and thus $x_{0} y_{0}=1$ and $(x \times y)_{1}(x \times y)_{2}=1$. Hence the above conclusion for these $x, y$ reads $1+\overline{\operatorname{det}(g)}=1+\operatorname{det}(g)$, whence $\operatorname{det}(g)=$ $\overline{\operatorname{det}(g)}=1$. Thus we showed that if $\lambda$ is a linear automorphism of $\mathcal{M}$, then $\lambda$ is of the form $\tilde{g}$ for some $g \in \mathrm{SL}(V)$.

Conversely, for every $g \in \mathrm{SL}(V)$, we define $\lambda:=\tilde{g}$ to be a linear map defined by equations (14) and (15). Then it follows from equations (17) and (18) that $m(x, y) \lambda=m(x g, y g)$ for all $x, y \in V$ with $[x] \neq[y]$. As $m(x, x) \lambda=\Delta(x) \lambda=$ $\sum_{i=0}^{2} \overline{x_{i}} \Delta_{i} \lambda=\sum_{i=0}^{2} \overline{x_{i}} \Delta\left(g_{i}\right)=\Delta(x g)$, the equation $m(x, y)=m(x g, y g)$ holds for all $x, y \in V$, and therefore $\lambda=\tilde{g}$ is in fact a linear automorphism of $\mathcal{M}$.

The same idea described in the second paragraph of the proof for Proposition 2.5 can also be used to find an automorphism which moves $A$.

Lemma 2.6. Let $\sigma$ be a linear bijection on $S^{2}(V)$ which fixes $\nabla_{2}$ and $\Delta_{2}$ and interchanges the pairs $\left(\nabla_{i}, \Delta_{i}\right)$ for $i=0$ and 1 . Then $\sigma$ is an automorphism 




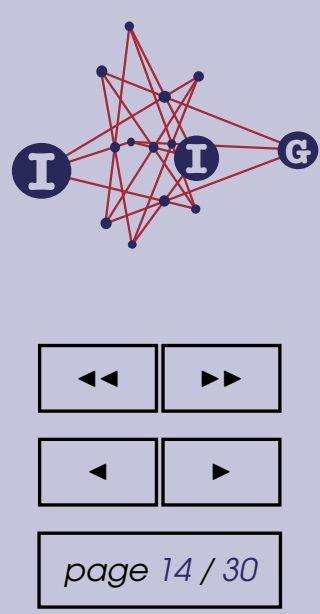

go back

full screen

close

quit

ACADEMIA

PRESS

Nin

$\widehat{\underline{\text { IIIIII }}}$

UNIVERSITEIT

GENT

$\{0,1,2\}), \bar{h}$ denotes the matrix whose $(a, i)$-entry is $\overline{h_{a i}}=h_{a i}^{2}$. Furthermore, $\iota(h)$ denotes the matrix whose $(a, i)$-entry is $h_{a j} h_{a k}$ for indices $j, k$ with $\{i, j, k\}=$ $\{0,1,2\}$. Thus $\iota(h)$ is the matrix obtained from $h$ by applying the operation $\iota$ introduced in Section 2.1 to all rows of $h$. With this notation, the matrix representing $\tilde{g}$ with respect to $\Delta_{i}, \nabla_{i}(i=0,1,2)$ is:

$$
\left(\begin{array}{cc}
\bar{g} & 0 \\
L(g) & { }^{t} g^{-1}
\end{array}\right), \quad \text { with } L(g):={ }^{t}\left(\iota\left({ }^{t} g\right)\right)+\iota\left({ }^{t}(\bar{g})^{-1}\right) .
$$

This is immediately obtained from equations (14) and (15), noticing the following facts: $\left(g_{b} \times g_{c}\right)_{i}=g_{b j} g_{c k}+g_{b k} g_{c j}(\{a, b, c\}=\{i, j, k\}=\{0,1,2\})$ is the determinant of the matrix obtained from $g$ by deleting the $a$-th row and the $i$-th column, whence it is the $(i, a)$-entry of the so called the adjugate matrix of $g$; the adjugate matrix of a matrix $g$ with $\operatorname{det}(g)=1$ coincides with $g^{-1}$. The above formula is convenient to calculate $\tilde{g}$ for a matrix $g \in \mathrm{SL}(V)$ specifically when $g_{i j} \neq 0$ for all $i, j \in\{0,1,2\}$. This also shows that the $L(\mathcal{M})_{A}$ (@ $\mathrm{SL}(V)$ )-module $S^{2}(V) / A$ is the Frobenius twist of the dual module to $A$. As the map sending $g \in \mathrm{SL}(V)$ to $\tilde{g} \in L(M)_{A}$ is an isomorphism, we should have $L(g h)=L(g) \bar{h}+\left({ }^{t} g^{-1}\right) L(h)$ for all $g, h \in \mathrm{SL}(V)$. This can be obtained from the following formula for any matrices $a, b$ of degree 3 , which can be verified by direct calculations:

$$
\iota(a b)=\bar{a} \iota(b)+\iota(a)\left({ }^{t} b^{-1}\right) .
$$

\subsection{Comments about the construction and automorphisms}

Del Fra [2] showed the uniqueness up to isomorphism of a 2-(projective) dimensional DHO over $\mathbb{F}_{4}$ with a certain condition, which is satisfied by the DHO $\mathcal{M}$. Nakagawa showed the uniqueness of a unitary DHO of rank 3 over $\mathbb{F}_{4}$ with ambient space $U$ of dimension 6 [6]. He also gives a precise description of all members of the Mathieu DHO, and showed that its full automorphism group contains the triple central cover of $M_{22}$ as a subgroup of index 2 .

Thanks to the above-mentioned result by Nakagawa, it follows from Proposition 2.2 that our DHO $\mathcal{M}$ is isomorphic to the Mathieu DHO. In fact, without much effort, we can verify that the above member $A$ and $A[v]$ of $\mathcal{M}$ are respectively identical to his member $A$ and $A[v]$, if we identify $\Delta_{i}$ and $\nabla_{i}(i=0,1,2)$ with $\mathbf{e}_{i}$ and $\mathbf{e}_{5-i}(i=0,1,2)$. Thus, in this sense, the construction above just gives another presentation of a well-known model of the Mathieu DHO. However, observe that our construction is simple and uniform, and that it makes easy to find the intersections of two given members (this is a bit messy for members of type $A[\alpha \theta, \alpha, 1]$ with notation in [6]). Furthermore, equation (8) shows that $\mathcal{M}$ is a deformation of the Veronesean DHO $\mathcal{V}_{3}\left(\mathbb{F}_{4}\right)$, because $x \times y(y \neq 0)$ is a typical vector in a member $V[y]$ of $\mathcal{V}_{3}\left(\mathbb{F}_{4}\right)$. 
Proposition 2.7 has been essentially shown in [6] as well. One advantage of our approach via the $\mathrm{DHO} \mathcal{M}$ is that we obtain rather explicit unitary matrices of degree 6 generating $L(\mathcal{M})$. These matrices will be effectively used to classify certain subspaces of $S^{2}(V)$ (see Section 3.4).

\section{Complements to the Mathieu DHO}

\subsection{Splitness of the Mathieu DHO}

We use the notation and convention in Section 2.1. Let $\mathcal{M}$ be the model of the Mathieu DHO constructed in Section 2.2. Recall that a complement to $\mathcal{M}$ is a subspace $Y$ of $S^{2}(V)$ with $S^{2}(V)=X \oplus Y$ for every member $X$ of $\mathcal{M}$. We shall interpret the existence of a complement in terms of some alternating semi-bilinear maps, and then show that $\mathcal{M}$ is of split type.

Lemma 3.1. Any complement to $\mathcal{M}$ coincides with

$$
Y(g):=\left\{\Delta(g(x, y))+\sum_{i=0}^{2}(x \times y)_{i} \nabla_{i} \mid x, y \in V\right\}
$$

for a map $g$ from $V \times V$ to $V$ which satisfies the following conditions.

(i) $g(x, x)=0, g(\alpha x, y)=\bar{\alpha} g(x, y), g\left(x+x^{\prime}, y\right)=g(x, y)+g\left(x^{\prime}, y\right)$ for all $x, x^{\prime}, y \in V$ and $\alpha \in \mathbb{F}_{4}$.

(ii) for every nonzero vector $a, b \in V$ with $[a] \neq[b]$,

$$
\sum_{i=0}^{2} \overline{(a \times b)_{i}} g\left(e_{j}, e_{k}\right) \neq \sum_{i=0}^{2}\left\{\overline{a_{i} b_{i}}+(a \times b)_{j}(a \times b)_{k}\right\} e_{i},
$$

where the indices $i, j, k$ follow the convention that $\{i, j, k\}=\{0,1,2\}$.

Proof. We first show that a 3-dimensional subspace $Y$ of $S^{2}(V)$ intersects $A=$ $\{\Delta(x) \mid x \in V\}$ trivially if and only if $Y=Y(g)$ for a map $g$ from $V \times V$ to $V$ satisfying condition (i) in Lemma. Assume that $Y$ is a subspace of $S^{2}(V)$ with $A \oplus Y=S^{2}(V)$. Then the vector $m(x, y) x, y \in V$ in equation (8) is uniquely written as $m(x, y)=\Delta(f(x, y))+\gamma(x, y)$ with $f(x, y) \in V, \gamma(x, y) \in Y$.

We denote by $\Delta(g(x, y))$ and $\nabla(x, y)$ respectively the sums of the diagonal and off-diagonal components of $\gamma(x, y)$. Then we have $\gamma(x, y)=\Delta(g(x, y))+$ $\nabla(x, y)$. In view of the diagonal and off-diagonal components of $m(x, y)=$ $\Delta(f(x, y)+g(x, y))+\nabla(x, y)$, we have

$$
\begin{aligned}
\nabla(x, y) & =\sum_{i=0}^{2}(x \times y)_{i} \nabla_{i}, \\
f(x, y)+g(x, y) & =\sum_{i=0}^{2}\left\{\overline{x_{i} y_{i}}+(x \times y)_{j}(x \times y)_{k}\right\} e_{i} .
\end{aligned}
$$


As $m(x, y)=m(y, x), m(x, x)=\Delta(x)$ and $m(\alpha x, y)=\alpha m(x, y)$ for all $x, y \in V$ and $\alpha \in \mathbb{F}_{4}$, we have

$$
\begin{array}{lll}
f(x, y)=f(y, x), & f(x, x)=x, & f(\alpha x, y)=\bar{\alpha} f(x, y) \\
g(x, y)=g(y, x), & g(x, x)=0, & g(\alpha x, y)=\bar{\alpha} g(x, y) .
\end{array}
$$

As $m\left(x+x^{\prime}, y\right)+m(x, y)+m\left(x^{\prime}, y\right)=\Delta(\delta y)$ with $\delta=\operatorname{det}\left(r\left(x, x^{\prime}, y\right)\right)$ for $x, x^{\prime}, y \in$ $V$ (see equation (11)),

$$
\Delta\left(f\left(x+x^{\prime}, y\right)+f(x, y)+f\left(x^{\prime}, y\right)+\delta y\right)=\gamma\left(x+x^{\prime}, y\right)+\gamma(x, y)+\gamma\left(x^{\prime}, y\right) .
$$

The left hand side of this equation lies in $A$, while the right hand side lies in $Y$. As $A \cap Y=\{0\}$, this implies that $f\left(x+x^{\prime}, y\right)=f(x, y)+f\left(x^{\prime}, y\right)+\delta\left(r\left(x, x^{\prime}, y\right)\right) y$ and $\gamma\left(x+x^{\prime}, y\right)=\gamma(x, y)+\gamma\left(x^{\prime}, y\right)$. As $\nabla(x, y)=\sum_{i=0}^{2}(x \times y)_{i} \nabla_{i}$ is bilinear, we have $g\left(x+x^{\prime}, y\right)=g(x, y)+g\left(x^{\prime}, y\right)$ for any $x, x^{\prime}, y \in V$. Thus $g$ satisfies the property (i) in Lemma. Furthermore, $Y$ coincides with

$$
Y(g):=\left\{\Delta(g(x, y))+\sum_{i=0}^{2}(x \times y)_{i} \nabla_{i} \mid x, y \in V\right\},
$$

because $Y(g)$ is a subset of $Y$ which forms a subspace of $S^{2}(V)$ by property (i) and it contains three independent vectors $\Delta\left(g\left(e_{j}, e_{k}\right)\right)+\nabla_{i}(i \in\{0,1,2\}=$ $\{i, j, k\})$.

Conversely, if $g$ is a map from $V \times V$ to $V$ having property (i), then $Y(g)$ defined in Lemma forms a subspace of $S^{2}(V)$, which is of dimension 3 in view of the off-diagonal parts. We have $Y(g) \cap A=\{0\}$, because if a vector $\Delta(g(x, y))+$ $\sum_{i=0}^{2}(x \times y)_{i} \nabla_{i}$ lies in $A$, then $x \times y=0$, and therefore $x=0$ or $y=\alpha x$ for some $\alpha \in \mathbb{F}_{4}$, which implies $g(x, y)=0$.

Thus in order to verify Lemma it suffices to show that $Y(g) \cap A[b]=\{0\}$ for any projective point $[b]$ in $\mathbf{P G}(V)$ if and only if $g$ satisfies the property (ii). There is a nonzero vector $b \in V$ such that $Y(g) \cap A[b] \neq\{0\}$ if and only if there are vectors $x, y, a \in V$ such that $a \neq 0$ and $[a] \neq[b]$ and $m(a, b)=\Delta(g(x, y))+$ $\sum_{i=0}^{2}(x \times y)_{i} \nabla_{i}$. Comparing the off-diagonal and diagonal components of the both sides of this equation, this is equivalent to the following conditions:

$$
a \times b=x \times y(\neq 0) \quad \text { and } \quad g(x, y)=\sum_{i=0}^{2}\left\{\overline{a_{i} b_{i}}+(a \times b)_{j}(a \times b)_{k}\right\} e_{i} .
$$

Then $x$ and $y$ are nonzero vectors lying in $\langle a, b\rangle=(a \times b)^{\perp}$, the hyperplane of $V$ perpendicular to $a \times b$ with respect to the dot product. Thus there are $\alpha, \beta, \gamma$ and $\delta$ in $\mathbb{F}_{4}$ such that $x=\alpha a+\beta b$ and $y=\gamma a+\delta b$. As $0 \neq a \times b=x \times y=$ $(\alpha \delta+\beta \gamma)(a \times b)$, we have $\alpha \delta+\beta \gamma=1$. As $g$ satisfies the property (i), we have $g(x, y)=\sum_{i=0} \overline{(x \times y)_{i}} g\left(e_{j}, g_{k}\right)=\sum_{i=0}^{2} \overline{(a \times b)_{i}} g\left(e_{j}, e_{k}\right)$. Thus there is no nonzero $b \in V$ with $Y(g) \cap A[b] \neq\{0\}$ if and only if the property (ii) holds. 




\subsection{DHO-sets for the Mathieu DHO}

In this section, we shall describe the DHO-set $\mathcal{L}_{A}(\mathcal{M} ; Y(g))$ for the DHO $\mathcal{M}$ with respect to the member $A$ and the complement $Y(g)$ for an alternating map $g$ defined in Proposition 3.2. We set $\Gamma_{i}:=\omega \Delta_{i}+\nabla_{i}$ for $i \in\{0,1,2\}$. Then $\Gamma_{i}(i=0,1,2)$ form a basis for the complement $Y(g)$. Notice that $\left(\Delta_{i}, \Gamma_{i^{\prime}}\right)=$ $\delta_{i, i^{\prime}}$ and $\left(\Gamma_{i}, \Gamma_{i^{\prime}}\right)=\delta_{i, i^{\prime}}$ for $i, i^{\prime} \in\{0,1,2\}$, where the symbol $\delta_{i, i^{\prime}}$ denotes the Kronecker's delta.

Take $[v] \in \mathbf{P G}(V)$. As $\nabla_{i}=\Gamma_{i}+\omega \Delta_{i}(i \in\{0,1,2\})$, a typical vector $m(x, v)=$ $\sum_{i=0}^{2}\left((x \times v)_{i} \nabla_{i}+z_{i} \Delta_{i}\right)$ in $A[v]$ (with $\left.z_{i}=x_{i} v_{i}+\overline{(x \times v)_{j}(x \times v)_{k}}\right)$ is written as $m(x, y)=\sum_{i=0}^{2}\left\{(x \times v)_{i} \Gamma_{i}+\left(\omega(x \times v)_{i}+z_{i}\right) \Delta_{i}\right\}$. Thus the decomposition of $m(x, v)$ in the direct sum $A \oplus Y(g)$ is given by $m(x, v)=a(x, v)+a(x, v) L[v]$, where

$$
\begin{aligned}
a(x, y) & =\sum_{i=0}^{2}\left(\omega(x \times v)_{i}+x_{i} v_{i}+\overline{(x \times v)_{j}(x \times v)_{k}}\right) \Delta_{i} \quad \text { and } \\
a(x, y) L[v] & =\sum_{i=0}^{2}(x \times v)_{i} \Gamma_{i} .
\end{aligned}
$$

We shall calculate an explicit form of $L[v]$ above as the matrix $M[v]$ representing $L[v]$ with respect to a basis $\left(\Delta_{i}\right)_{i=0}^{2}$ for $A$ and a basis $\left(\Gamma_{i}\right)_{i=0}^{2}$ for $Y(g)$. We set $M[0]=0$ and $\tilde{V}:=\{[0]\} \cup \mathbf{P G}(V)$. By the discussions in the previous section, $M[v]+M[w]$ is of rank 2 for any distinct $[v],[w]$ in $\tilde{V}$. If the kernel of $M[v]+M[w]$ is denoted by $\kappa([v],[w])$, then the map sending $[w]$ to $\kappa([v],[w])$ is a bijection on $\tilde{V} \backslash\{[v]\}$. This collection of matrices $M[v]$ for $[v] \in \tilde{V}$ also satisfy the following property:

Lemma 3.3. For each $[v] \in \tilde{V}$, the matrix $U[v]:=M[v]+I$, with the identity matrix $I$, is a unitary matrix, in the sense that $U[v]^{t} \overline{(U[v])}=I$.

Proof. As the claim is trivial for $[v]=[0]$, we may assume that $[v]$ lies in $\mathbf{P G}(V)$. Then $0=(m(x, v), m(y, v))$ for any $x, y \in V$ by Proposition 2.2(iv). Putting $m(z, v)=a(z, v)+a(z, v) L[v]$ for $z \in\{x, y\}$, we have

$$
0=(a(x, v), a(y, v) L[v])+(a(x, v) L[v], a(y, v))+(a(x, v) L[v], a(y, v) L[v]) .
$$

Using the matrix $M[v]=\left(\mu_{i j}\right)$ representing $L[v]$, this equation for $x, y$ with $a(x, v)=\Delta_{i}, a(y, v)=\Delta_{i^{\prime}}$ reads $0=\left(\Delta_{i}, \sum_{j=0}^{2} \mu_{i^{\prime} j} \Gamma_{j}\right)+\left(\sum_{j=0}^{2} \mu_{i j} \Gamma_{j}, \Delta_{i^{\prime}}\right)+$ $\left(\sum_{j=0}^{2} \mu_{i j} \Gamma_{j}, \sum_{j=0}^{2} \mu_{i^{\prime} j} \Gamma_{j}\right)=\overline{\mu_{i^{\prime} i}}+\mu_{i i^{\prime}}+\sum_{j=0}^{2} \mu_{i j} \overline{\mu_{i^{\prime} j}}$. This is equivalent to the condition that $M[v]+{ }^{t} \overline{M[v]}+M[v]^{t} \overline{M[v]}=0$, or equivalently, to the condition that $(M[v]+I)^{t}(M[v]+I)=I$.

For short, we set $a(x, v)=\sum_{i=0}^{2} y_{i} \Delta_{i}$ and $a(x, v) L[v]=\sum_{i=0}^{2} z_{i} \Gamma_{i}$. Thus

$$
y_{i}:=x_{i} v_{i}+\omega(x \times v)_{i}+\overline{(x \times v)_{j}(x \times v)_{k}}, \quad z_{i}:=(x \times v)_{i} .
$$





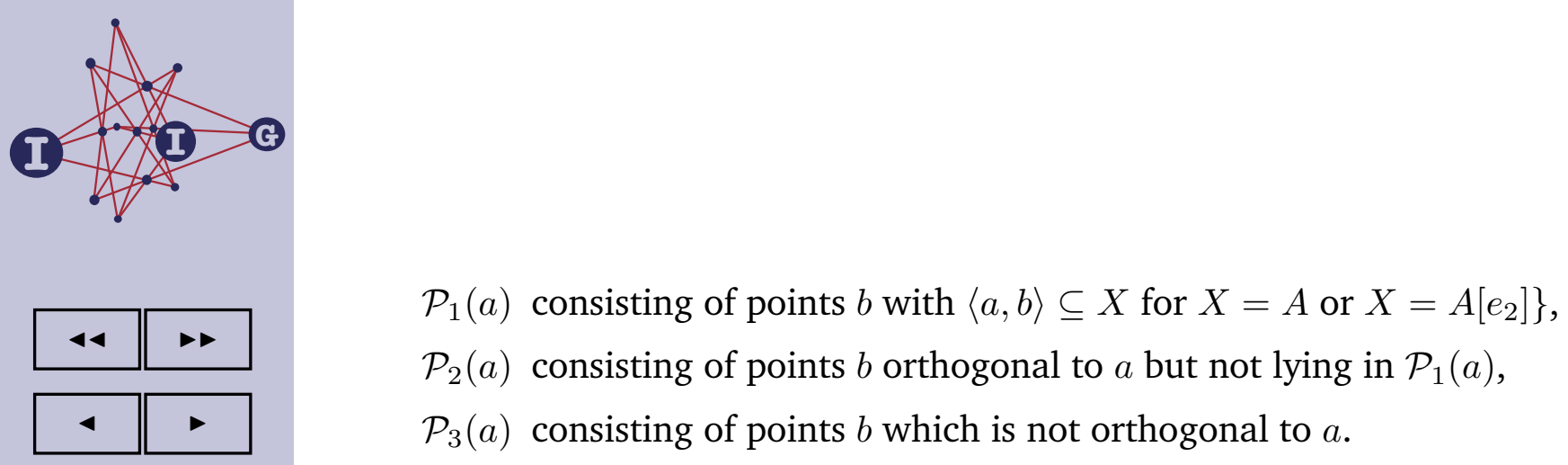

Using the triple transitivity of $L(\mathcal{M})$ on the members of $\mathcal{M}$, we can see that $\mathcal{P}_{i}(a)(i=0,1,2)$ are orbits under the stabilizer $L(\mathcal{M})_{a}$. For $\mathcal{P}_{1}(a)$, this follows from the doubly transitivity of $\mathrm{SL}(V)$ on the set of projective points in $\mathbf{P G}(V)$ and the existence of the automorphism $\sigma$ in $L(\mathcal{M})_{a}$ exchanging two members $A$ and $A\left[e_{2}\right]$ containing $a$. Now take a point $b$ in $\mathcal{P}$ not lying on $A$ nor $A\left[e_{2}\right]$. By the triple transitivity of $L(\mathcal{M})$ on the members of $\mathcal{M}$, there is a linear automorphism $\lambda$ of $\mathcal{M}$ stabilizing $A$ and $A\left[e_{2}\right]$ (and hence fixing $a=A \cap A\left[e_{2}\right]$ ) such that $b \lambda \in$ $A\left[e_{1}\right]$. If $b$ lies in $\mathcal{P}_{2}(a)$ (resp. $\mathcal{P}_{3}(a)$ ), $b \lambda$ is orthogonal (resp. not orthogonal) to $a$, because $\lambda$ preserves the unitary form (, ) by Lemma 2.3. Then, if $b \in \mathcal{P}_{2}(a)$, $b \lambda$ lies in the line $l:=A\left[e_{1}\right] \cap a^{\perp}=\left\langle\Delta_{1}, \nabla_{0}\right\rangle$. Notice that the stabilizer of the line $l$ in $L(\mathcal{M})_{A\left[e_{1}\right]} \cong \mathrm{SL}(V)$ (Proposition 2.5) induces $A_{5}$ on the five points on $l$. Thus the stabilizer $G$ of $l$ and two points $\left\langle\Delta_{1}\right\rangle$ and $\left\langle\nabla_{0}\right\rangle$ is transitive on the remaining three points, one of which is $b \lambda$. Notice that $G$ stabilizes the unique members $A$ and $A\left[e_{2}\right]$ of $\mathcal{M} \backslash\left\{A\left[e_{1}\right]\right\}$ containing $\left\langle\Delta_{1}\right\rangle$ and $\left\langle\nabla_{0}\right\rangle$ respectively, whence fixes $a=A \cap A\left[e_{2}\right]$. Thus the transitivity of $L(\mathcal{M})_{a}$ on $\mathcal{P}_{2}(a)$ is established. If $b \in \mathcal{P}_{3}(a)$, then $b \lambda$ is one of the 16 points in $A\left[e_{1}\right]$ outside the line $l$, on which the point-wise stabilizer $K$ of $l$ in $L(\mathcal{M})_{A\left[e_{1}\right]}$ acts regularly. As $K$ fixes $\left\langle\Delta_{1}\right\rangle$ and $\left\langle\nabla_{0}\right\rangle, K$ lies in $L(\mathcal{M})_{a}$. This established the transitivity of $L(\mathcal{M})_{a}$ on $\mathcal{P}_{3}(a)$. We have $\left|\mathcal{P}_{2}(a)\right|=20 \cdot 3 / 2=30$, because each point in $\mathcal{P}_{2}(a)$ lies in two members in $\mathcal{M} \backslash\left\{A, A\left[e_{2}\right]\right\}$ and each member $X$ in $\mathcal{M} \backslash\left\{A, A\left[e_{2}\right]\right\}$ contains the line $X \cap a^{\perp}$ consisting of three points in $\mathcal{P}_{2}(a)$. Furthermore, $\left|\mathcal{P}_{1}(a)\right|=2 \times 20=40$ and $\left|\mathcal{P}_{3}(a)\right|=|\mathcal{P}|-1-40-30=160$. A point $\left\langle\Delta_{2}+\alpha\left(\Delta_{1}+\nabla_{0}\right)\right\rangle\left(\alpha \in \mathbb{F}_{4}\right)$ on the line through $a=\left\langle\Delta_{2}\right\rangle$ and $b=\left\langle\Delta_{1}+\nabla_{0}\right\rangle$ $\left(\in \mathcal{P}_{2}(a)\right)$ lies in $\mathcal{P}$ if and only if $\Delta_{2}+\alpha\left(\Delta_{1}+\nabla_{0}\right)=m(x, y)$ for some $x, y \in V$. Thus we have $(x \times y)_{0}=\alpha,(x \times y)_{1}=(x \times y)_{2}=0$, and $x_{0} y_{0}=0, x_{1} y_{1}=\alpha$ and $x_{2} y_{2}=1$. It can be verified that this holds exactly when $\alpha=1$. Thus $\left\langle\Delta_{2}+\Delta_{1}+\nabla_{0}\right\rangle$ is the unique point in $\mathcal{P}$ on the line through $a$ and $b$ other than $a$ and $b$. If $b=\left\langle\nabla_{2}\right\rangle \in \mathcal{P}_{3}(a)$, it is also easy to verify that the third isotropic point $\left\langle\Delta_{2}+\nabla_{2}\right\rangle$ is not in $\mathcal{P}$. Summarizing, we have:

Lemma 3.6. For each $a \in \mathcal{P}$, there are exactly 4 orbits on $\mathcal{P}$ under the action of the stabilizer $L(\mathcal{M})_{a}$ of $a$ in $L(\mathcal{M})$. Three obits $\mathcal{P}_{i}(a)(i=1,2,3)$ except the trivial orbit $\{a\}$ are described as follows.

(i) $\mathcal{P}_{1}(a)$ consists of $b$ in $\mathcal{P}$ such that the line through $a$ and $b$ is contained in some members of $\mathcal{M}$. We have $\left|\mathcal{P}_{1}(a)\right|=40$. For $b \in \mathcal{P}_{1}(a)$, the isotropic line $\langle a, b\rangle$ consists of 5 isotropic points in $\mathcal{P}$.

(ii) $\mathcal{P}_{2}(a)$ consists of $b$ in $\mathcal{P}$ orthogonal to a (with respect to the unitary form 





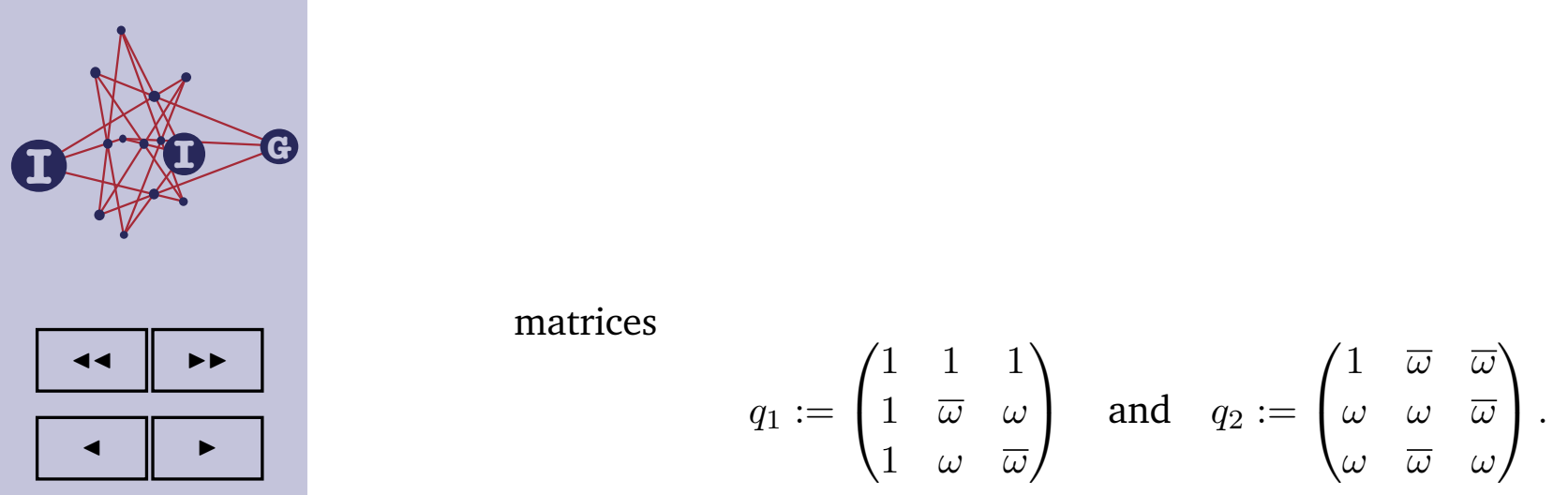

It is easy to see that $\left(q_{1}\right)^{2}=\left(q_{2}\right)^{2}=\left(e_{1}, e_{2}\right)$ and $q_{1}^{q_{2}}=q_{1}^{-1}$, and hence $\left\langle q_{1}, q_{2}\right\rangle \cong Q_{8}$. Furthermore, we can verify that $t_{1}^{q_{1}}=t_{2}, t_{2}^{q_{1}}=t_{1}, t_{1}^{q_{2}}=$ $t_{1} t_{2}\left(\omega I_{3}\right)^{2}$ and $t_{2}^{q_{2}}=t_{1} t_{2}^{-1}\left(\omega I_{3}\right)$. Thus $\left\langle q_{1}, q_{2}\right\rangle$ normalizes $T$. From Proposition 2.5, we can obtain the following linear maps $\tilde{q}_{1}$ and $\tilde{q}_{2}$ in $L(\mathcal{M})$ (stabilizing $A$ ) with some calculations:

$$
\tilde{q}_{1}:=\left(\begin{array}{cc}
\overline{q_{1}} & 0 \\
0 & \overline{q_{1}}
\end{array}\right) \quad \text { and } \quad \tilde{q}_{2}:=\left(\begin{array}{cc}
\overline{q_{2}} & 0 \\
\overline{q_{2}} & \overline{q_{2}}
\end{array}\right)
$$

where $\bar{A}:=\left(\overline{a_{i j}}\right)$ for $A=\left(a_{i j}\right)$. Thus $\tilde{Q}:=\left\langle\tilde{q}_{1}, \tilde{q}_{2}\right\rangle\left(\cong Q_{8}\right)$ is a Sylow 2-subgroup of the normalizer of $\tilde{T}$ in $L(\mathcal{M})$.

As we remarked above, the stabilizer of the plane $Y_{0}$ in $L(\mathcal{M})$ is a subgroup of the normalizer of $\tilde{T}$ (which is $\tilde{T} \tilde{Q}$ ) containing $\tilde{Q}$. It is straightforward to see that that $\tilde{q}_{1}$ preserves $Y_{0}$ but $\tilde{q}_{2}$ does not. In fact, we have $a_{0} \tilde{q}_{1}=$ $a_{0}+a_{1}+a_{2}, a_{1} \tilde{q}_{1}=a_{0}+\bar{\omega} a_{1}+\omega a_{2}$ and $a_{2} \tilde{q}_{1}=a_{0}+\omega a_{1}+\bar{\omega} a_{2}$; but $a_{1} \tilde{q}_{2}=\left(\Delta_{0}+\omega \nabla_{0}\right)+\left(\Delta_{1}+\omega \nabla_{1}\right)+\bar{\omega}\left(\Delta_{2}+\omega \nabla_{2}\right)$, which is not contained in $Y_{0}$. (In fact, for the semi-linear automorphism $\iota$ on $S^{2}(V)$ sending a vector $\sum_{i=0}^{2}\left(x_{i} \Delta_{i}+y_{i} \nabla_{i}\right)$ to $\sum_{i=0}^{2}\left(\overline{x_{i}} \Delta_{i}+\overline{y_{i}} \nabla_{i}\right)$, we can verify that $\iota$ is an automorphism of $\mathcal{M}$ and that $\tilde{q}_{2} \iota$ preserves $Y_{0}$.) As $\tilde{T}\left\langle\tilde{q}_{1}\right\rangle$ is a maximal subgroup of the normalizer $\tilde{T} \tilde{Q}$, we conclude that the stabilizer of $Y_{0}$ in the linear automorphism group $L(\mathcal{M})$ coincides with $\tilde{T}\left\langle\tilde{q}_{1}\right\rangle\left(\cong 3_{+}^{1+2}: Z_{4}\right)$.

(3) As $a:=\left\langle\Delta_{0}\right\rangle$ and $b:=\left\langle\nabla_{0}\right\rangle$ are the only points of $\mathcal{P}$ contained in $Y_{2}$ by claim (1), the stabilizer $L(\mathcal{M})_{Y_{2}}$ of the plane $Y_{2}$ in $L(\mathcal{M})$ is a subgroup of the setwise stabilizer of $\{a, b\}$ in $L(\mathcal{M})$. As $a$ and $b$ are not orthogonal, $b$ lies in the suborbit $\mathcal{P}_{3}(a)$ in Lemma 3.6. Then the poitwise stabilizer $L(\mathcal{M})_{a, b}$ is a subgroup of $L(\mathcal{M})_{a}\left(\cong 3.2^{4} A_{5} .2\right)$ of index $\left|\mathcal{P}_{3}(a)\right|=160$, so that $\left|L(\mathcal{M})_{a, b}\right|=2^{2} .3^{2}$. As the automorphism $\sigma$ in Lemma 2.6 fixes $\Delta_{0}$ and $\nabla_{0}$ but exchanges $\Delta_{2}$ and $\nabla_{2}$, we conclude that the set wise stabilizer of $\{a, b\}$ in $L(\mathcal{M})$ coincides with the group $\left\langle\omega I_{6}, \tilde{t}_{2}, \tilde{q}_{1}^{2}, \tilde{t}_{1} \sigma \tilde{t}_{1}^{-1}\right\rangle\langle\sigma\rangle$, where $\tilde{t}_{i}$ $(i=1,2)$ are the automorphisms of $\mathcal{M}$ appeared in the proof of claim (2). It is straightforward to determine the stabilizer of $Y_{2}$ in $L(\mathcal{M})$ coincides with $\left\langle\omega I_{6}, \tilde{t}_{2}, \sigma\right\rangle\left(\cong E_{9} \times 2\right)$, working inside this small group.

Finally the following lemma establishes Proposition 3.5, because the set of complements coincides with $\mathcal{S}(0)$ by Lemma 3.8 .

Lemma 3.11. We have $\mathcal{S}=\mathcal{S}(0) \cup \mathcal{S}(2) \cup \mathcal{S}(4)$, where $\mathcal{S}(i)$ coincides with the orbit under $L(\mathcal{M})$ containing $Y_{i}$ for every $i \in\{0,2\}$. 

Let $\mathcal{S}$ be a proper subDHO of $\mathcal{M}$. Since $L(\mathcal{M})$ is transitive on the members of $\mathcal{M}$ and the stabilizer of $A$ in $L(\mathcal{M})$ is transitive on the hyperplanes of $A$, there is an automorphism $\alpha$ of $\mathcal{M}$ such that $\mathcal{S}^{\alpha}$ contains $\Delta(W):=\{\Delta(x) \mid x \in W\}$ as a member, where $W$ is a hyperplane of $V$ spanned by $e_{0}$ and $e_{1}$. For each projective point $[v]$ in $\mathbf{P G}(W)$, we denote by $S[v]$ the unique member of $\mathcal{S}$ distinct from $\Delta(W)$ containing $[v]$. Then $S[v]$ is a hyperplane of $A[v]$. As $\mathcal{S}$ is a DHO, $S[v] \cap S[w]$ is a 1-dimensional subspace for distinct projective points $[v]$ and $[w]$ in $\mathbf{P G}(B)$. On the other hand, $A[v] \cap A[w]$ is a 1-dimensional subspace of $U$ spanned by $m(v, w)$. Thus we have $S[v] \cap S[w]=A[v] \cap A[w]$. Notice that $v \times w=\left(v_{0} w_{1}+w_{0} v_{1}\right) e_{2}$ for $v, w \in W$, so that $m(v, w)=v \otimes w+\Delta(\iota(v \times w))=$ $v \otimes w$. As a 2-dimensional subspace $S[v]$ is spanned by $\Delta(v)$ and $m(v, w)=$ $v \otimes w$, we conclude that $S[v]=\{v \otimes x \mid x \in W\}$ for each $0 \neq v \in W$. Thus $\mathcal{S}^{\alpha}=\{\Delta(W)\} \cup\{S[v] \mid[v] \in \mathbf{P G}(W)\}$ coincides with the Veronesean DHO $\mathcal{V}_{2}\left(\mathbb{F}_{4}\right)$ with ambient space $S^{2}(W)$.

As the ambient space of any DHO of rank 3 over $\mathbb{F}_{4}$ has dimension at most 6 by, e.g., [8, Theorem 1(i)], any cover of $\mathcal{M}$ is isomorphic to $\mathcal{M}$. Finally, there is no proper quotient of $\mathcal{M}$, by the following observation to any DHO of polar type, applied to the unitary DHO $\mathcal{M}$.

Lemma 4.1. Let $\mathcal{S}$ be a DHO of rank $n$ over $\mathbb{F}_{q}$ with ambient space $A$ of dimension $2 n$ equipped with a non-degenerate alternating, quadratic or hermitian form $f$, in which any member of $\mathcal{S}$ is totally isotropic with respect to $f$. Then there is no proper quotient of $\mathcal{S}$.

Proof. Suppose there is a proper quotient $\overline{\mathcal{S}}$ of $\mathcal{S}$. Let $\pi$ be a covering map from $\mathcal{S}$ to $\overline{\mathcal{S}}$. As the ambient space of a DHO of rank $n$ is at least $2 n-1$, the kernel $K$ of a semilinear map $\pi$ is of dimension 1 . Let $k$ be a nonzero vector of $K$. Recall that $K \cap(X+Y)=\{0\}$ for any distinct member $X$ and $Y$ of $\mathcal{S}$ by [8, Proposition 13].

On the other hand, as $f$ is non-degenerate, $k^{\perp}:=\{v \in A \mid f(v, k)=0\}$ is a hyperplane of $A$. As $n>1$, the intersection $k^{\perp} \cap X$ for any member $X$ of $\mathcal{S}$ is of dimension at least 1 . Thus there is a nonzero vector $x$ of $X$ such that $f(x, k)=0$. Let $Y$ be the unique member of $\mathcal{S} \backslash\{X\}$ containing $x$. Then $X+Y=x^{\perp}$, as $X+Y$ is a hyperplane of $A$ containing isotropic subspaces $X$ and $Y$. As $k \in x^{\perp}$, this contradicts the fact that $K \cap(X+Y)=\{0\}$.

\section{Summarizing, we have}

Proposition 4.2. Any proper subDHO of $\mathcal{M}$ is conjugate under the action of $\operatorname{Aut}(\mathcal{M})$ to the Veronesean DHO $\mathcal{V}_{2}\left(\mathbb{F}_{4}\right)$ with ambient space $S^{2}(W)$ of dimension 3 , where $W$ is a hyperplane of $V$ spanned by $e_{0}$ and $e_{1}$. There is no proper cover nor proper quotient of $\mathcal{M}$. 


\section{References}

[1] J. H. Conway, R. T. Curtis, S. P. Norton, R. A. Parker and W. A. Wilson, Atlas of Finite Groups, Clarendon press, Oxford, 1985.

[2] A. Del Fra, On $d$-dimensional dual hyperovals, Geom. Dedicata 79 (2000), 157-178.

[3] U. Dempwolff, Dimensional doubly dual hyperovals and bent functions, Innov. Incidence Geom. 13 (2014), 149-178.

[4] W. Jónsson and J. McKay, More about the Mathieu group $M_{22}$, Canad. J. Math. 28 (1976), 929-937.

[5] C. Huybrechts and A. Pasini, Flag-transitive extensions of dual affine spaces, Beitr. Algebra Geom. 40 (1999), 503-532.

[6] N. Nakagawa, On 2-dimensional dual hyperovals of polar type, Util. Math. 76 (2008), 101-114.

[7] H. Taniguchi and S. Yoshiara, A unified description of some simply connected dual hyperovals, European J. Combin., 36 (2014), 143-150.

[8] S. Yoshiara, Ambient spaces of dimensional dual arcs, J. Algebraic Combin. 19 (2004), 5-23.

[9] _ Dimensional dual arcs-a survey, pp.247-266, in: Finite Geometries, Groups, and Computation, eds. A.Hulpke, B.Liebler, T.Penttila, and A.Seress, Walter de Gruyter, Berlin-New York, 2006.

[10] Disjoint unions of dimensional dual hyperovals, Innov. Incidence Geom. 14 (2015), 43-76.

[11] _ Splitness of the Veronesean and the Taniguchi dual hyperovals, manuscript.

Satoshi Yoshiara

Department of Mathematics,, TOKyo Woman's Christian University,, 2-6-1 Zempukuji, SUGINAMI-KU, TOKYO, , 167-8585 JAPAN

e-mail: yoshiara@lab.twcu.ac.jp 